\title{
The Avalanche Photodiode - A Promising Low Light Level Detector for Astronomical Photometry
}

\author{
G. Śzécésnyi-Nagy \\ Eötövs University of Budapest, Department of Astronomy, \\ H-1083 Budapest, Ludovika tr 2. Hungary
}

\begin{abstract}
Astronomical photometry like other scientific methods is always forced to search for up-to-the-minute instruments and the most sophisticated devices. The photomultiplier tube (PMT) is the most widely used photon detector offering high sensitivity and gain with acceptable quantum efficiency and spectral range but it also has some disadvantages. During the past few decades all of these parameters have been improved and it seems likely that possibilities for their continued development are exhausted.

In the meantime, technical development - especially micro electronics and semiconductor technology - has produced a new detector, the avalanche photodiode (APD). The APD which is often referred to as a solid-state PMT is just at the start of its career and we may expect its rapid perfection. Although properties of APDs were first discussed almost twenty years ago their mass production did not start until 1990. Early silicon APDs had very limited dimensions compared to PMTs but unfortunately this also implied that their photosensitive area was less than $0.25 \mathrm{~mm}$. One more year of development has been enough to increase the photon collecting surface by a factor of 1000 and APDs may soon become possible rivals of PMTs.

Following a brief description of the new device and discussion of its operation a comparison of photodetector performance will be given in order to draw attention to those parameters which are of great promise. Those sections of astronomical photometry which are ready to benefit from the suggested testing and use of this kind of photon detector will be highlighted.
\end{abstract}

1. Why do we need a solid-state photon detector with an internal gain mechanism?

At ultra-low light levels the electric current induced by incoming photons in the detector is definitely lower than the noise of any electronic amplifier connected to it. In order to be able to measure accurately the photon flux of very faint sources we need a detector which employs some kind of internal gain mechanism. Most generally this is internal multiplication or avalanching (multiplication of electrons produced by a photoemissive element or photocathode). 
In spite of practical drawbacks, vacuum photomultiplier tubes (PMTs) have long been the most widely used photon detectors for high accuracy astronomical photometry. But nowadays the replacement of vacuum or low-pressure gas tube devices with solid-state detectors is a general trend which seems to be irresistible. The mass production of semiconductor chips and other photonic circuits and sensors will probably result soon in a performance/price ratio superior to that of PMTs which are very complicated and delicate products of state-of-the-art technology and craftsmanship. Although I am confident that the PMT contributed more than anything else to the spectacular development of high-precision photoelectric photometry, it is also evident that the good old tubes must have passed the culmination of their career and that astronomers specialized in photometry have to consider more modern and simple detectors for the photometers of the next century. The avalanche photodiode (APD) with its internal gain mechanism is already competitive with PMTs in many aspects and we can be optimistic about its future.

\section{Principle of operation of APDs}

In these devices which are usually made of germanium or silicon the incoming photons produce excess carriers in the active (PN or PIN) junction. It is crucial that each photon whose energy exceeds the bandgap of the semiconductor material has the ability to produce one electron-hole pair and consequently may affect the overall junction potential which can be measured.

In a typical photodiode the photocurrent is independent of the applied bias and its value gives immediate information on the incoming flux. Typical diodes are linear over eight decades of incident photon flux (Eccles et al.1983) and this is their most promising characteristic. On the contrary, the magnitude of the applied bias voltage affects the speed of response of the device. Large reverse bias causes higher acceleration of the minority carriers across the junction, reduces their transit time and increases the response speed.

In a typical APD hundreds or thousands of volts is applied to the photo-diode as reverse bias. Consequently each electron generated in the $\mathrm{P}$ layer is accelerated by this enormous potential field to considerable energies as it passes the diode. Silicon has a bandgap of only $3.6 \mathrm{eV}$ so in a SiAPD each photo-electron has enough kinetic energy to excite several hundred free electrons by collisions in an avalanche effect. Carrier production avalanches because any new secondary electrons are accelerated by the high field too. Thus each infalling photon causes 100-1000 charge carriers to flow across the junction.

It is evident even from this brief description that the APD is a theoretically complex device that combines photon detection with electrical amplification (gain) in a simple monolithic solid-state structure. Consequently APDs in principle are similar to PMTs while being much simpler (two wires only, no glass vacuum tube, no dynodes, no voltage divider etc.), smaller, lighter, more compact, more rugged and immune to magnetic fields. An extra advantage of an APD over a PMT is that the former uses a single high voltage which draws only the photocurrent whereas PMTs 
need $10-20$ various voltage levels and they draw anode currents 20 times larger.

\section{Recent developments in APD technology}

While the APD has been recognized as a possible replacement for the PMT, until recently mass production has proved possible only for devices of small dimensions (diameters less than $1 \mathrm{~mm}$ ). These small area APDs (SAAPDs) are not yet competitive with PMTs in low light level photon detection in astronomy.

One of the most serious problems with APDs has been the small size of their light sensitive area (Smith 1992). SAAPDs have a break down voltage of only some hundreds of volts which is insufficient to reach high enough gain for low light level photon flux determination. But a newcomer to the field, Advanced Photonix, has successfully developed large-area APDs (LAAPDs) that can satisfy the needs of many applications currently using PMTs (Noble 1991). The new LAAPDs combine the benefits of solid-state photodiodes with many of the performance characteristics of a typical PMT. They provide high internal gain as a consequence of the much higher reverse voltage which can be applied because the break down voltage of these devices is about $2.5 \mathrm{kV}$ (Saloff \& Madden 1992). Using transmutated doped silicon and a proprietary edge treatment this firm now offers LAAPDs with diameters larger than 0.5 inch, capable of operating at voltages in excess of $2 \mathrm{kV}$. It is important too that the typical dark current of the device at $2.4 \mathrm{kV}$ reverse bias is only $200 \mathrm{nA}$ (Advanced Photonix 1992).

LAAPDs with much larger active areas than traditional APDs and with a close functional similarities to PMTs and photodiodes offer a real alternative to these devices and require little or no system redesign while significantly improving the performance/cost ratio. Furthermore using APDs in a proper housing and electric environment offers overall reduction in system size, weight and complexity, improved ruggedness, reliability and longevity. In addition they have an enormous dynamic range with a perfectly linear response from a few hundred photons to $10 \mathrm{~mW}$ incident intensity. They are immune to damage by overload and do not have the long settling times typical of PMTs (up to 100 hours - THORN EMI Electron Tubes Ltd. 1986).

LAAPDs of Advanced Photonix typically use only $5 \%$ of the current than an equivalent PMT and operate from a single high voltage. Another benefit of these solid-state photon detectors is that they are immune to the effects of stray fields for which they require no shielding at all (Saloff \& Madden 1992).

The maximum gain that has been reached by LAAPDs so far is considerably lower than that of a PMT used in astronomical photometers but it is not unduly optimistic to expect the appearance of SiAPDs 10-100 times more effective even in this decade. However, for ultra low light level applications like single photon counting, sophisticated PMTs remain the first-choice at least for five more years.

\section{Spectral sensitivity and responsivity of the APDs}

The spectral sensitivity of the photodiodes is affected by two phenomena. Reflec- 
tion of the infalling photons from the surfaces of the semiconductor and its housing or window and absorption of the radiation by the material of the window fix the short-wavelength limit of the sensitivity range. On the other hand the bandgap of the raw material of the APD determines the long wavelength threshold. In the case of commercially available detectors UV-sensitivity is very low but may be extended theoretically to $200 \mathrm{~nm}$ and over practically the whole of the astronomically interesting (ground-based) UV-range to $290 \mathrm{~nm}$, using proper window materials and/or phosphorescent coatings. For instance the UV-to-visible converter of Princeton Instruments is able to increase the quantum efficiency of silicon CCDs in the 250-400 $\mathrm{nm}$ range by a factor of 20 to 100 (Princeton Instruments Inc. 1991).

The IR-sensitivity of a photodiode is fixed by the band structure of its semiconductor material which depends on the temperature of the device. At room temperature silicon is capable of detecting photons with wavelengths below $1100 \mathrm{~nm}$ and germanium - the other popular raw material of photodiodes - is able to convert photons with wavelengths up to $1820 \mathrm{~nm}$ into carriers.

Earlier versions of SiAPDs had a spectral response range from $400 \mathrm{~nm}$ to $950 \mathrm{~nm}$ with a peak response at $800 \mathrm{~nm}$. At this wavelength their typical peak responsivity is $150 \mathrm{~A} / \mathrm{W}$. (Responsivity $R$ is the ratio of the photocurrent generated for every watt of incident light power with units of $\mathrm{A} / \mathrm{W}$ or $\mathrm{mA} / \mathrm{mW}$. It is the preferred measure of a photodiode's response to light.) Normally responsivity ranges between $50 \mathrm{~A} / \mathrm{W}$ and $150 \mathrm{~A} / \mathrm{W}$

The silicon photodiode response is usually linear within a few tenths of a percent from the minimum detectable incident light power up to several milliwatts and its linearity improves with increasing applied reverse bias and decreasing effective load resistance. Average SiAPDs normally have a dynamic range exceeding 10 million while the best devices give a linear response in a range at least ten times greater.

For photometrists who are more familiar with the quantum efficiency $(Q . E$. or $Q E)$ of their photon detectors a similar feature, the external quantum efficiency $(E Q E)$ can be defined as the percentage of incident power which results in an electric current which flows when an external load is connected to the photodiode. It can be calculated with the following formula:

$$
E Q E=123.96 R / L
$$

where $E Q E$ is the percentage of external quantum efficiency, $R$ is the photodiode responsivity (measured in amperes/watt) and $L$ is the wavelength of the incident radiation (in microns).

Typical values of quantum efficiency range from $50 \%$ to $95 \%$ depending on the wavelength of the incident light and the type of photodiode. $E Q E$ is always less than unity because of reflection losses at all wavelengths, surface loss mechanisms at VUV wavelengths and poor absorption of photons in the IR spectral range. Since at very low light levels the optimal exploitation of the incoming photons is an absolute necessity their $Q E$ is probably the most important feature of photodetectors. The table gives $E Q E$ and $Q E$ values for SiAPDs and PMTs respectively from $400 \mathrm{~nm}$ 
to $1100 \mathrm{~nm}$ (after MacGregor 1991 and THORN EMI Electron Tubes Ltd 1986). It is to be noted that typical PMT photocathodes have peak $Q E$ in the UV-range at about $380 \mathrm{~nm}$ while an SiAPD's $E Q E$ peaks in the near IR at about $850 \mathrm{~nm}$. The fourth column gives the values of the ratio $E$ in favour of SiAPDs.

\begin{tabular}{rrrr} 
Wavelength & SiAPD EQE & PMT $Q E$ & $E=E Q E / Q E$ \\
$(\mathrm{~nm})$ & $(\%)$ & $(\%)$ & \\
400 & 25 & 27 & 0.926 \\
400 & 25 & 27 & 0.926 \\
500 & 80 & 22 & 3.6 \\
600 & 85 & 10 & 8.5 \\
\hline 700 & 90 & 5 & 18 \\
800 & $>90$ & 2 & $>45$ \\
\hline 900 & 60 & $<0.5$ & $>120$ \\
1000 & 45 & $<0.3$ & $>150$ \\
1100 & 25 & $<0.1$ & $>250$
\end{tabular}

Values in the column of PMT $Q E$ at any wavelength refer to the highest $Q E$ of the following three cathode types: Bi-alkali, S1 or S20.

It is evident from the table that SiAPDs should soon replace PMTs in the red-IR spectral range. In the yellow (visual) and orange colours the excess factor is at least an order of magnitude lower than that needed to compensate for the momentarily (and temporarily?!) much higher gain of photomultipliers. Nevertheless the quick and unprecedented development of the semiconductor technology and its products during the last decades predicts the possible and effective perfection of APDs. While the internal gain of these devices will be increased by a factor of 100 , their overall signal will exceed that of the best PMTs over a much wider spectral range. And this is not a dream!

A team at EG\&G by operating with low dark-count (small active area) APDs above the breakdown voltage has been able to achieve avalanche gains exceeding one million and producing current pulses that have been easily detected (MacGregor 1991 and Noble 1991). It means that APDs could be used even in photon-counting applications (at present only if they have a diameter $\leq 1 \mathrm{~mm}$ ) provided that their bias and temperature are strictly controlled.

In view of the low ultraviolet quantum efficiency of APDs, the task of finding the best window materials and most effective $U V$-to-visual or $U V$-to-NIR converting layers is urgent. As it was mentioned, the efficiency of photo-electron production can be increased by a factor of up to 100 in this way.

It is time to mention some drawbacks of APDs. Dark current - an essential phenomenon which may limit the ultra low light applications of photodetectors - exceeds the acceptable level and it is higher in the case of LAAPDs which are otherwise more promising for astrophotometry. Another serious problem is the high temperature sensitivity of photodiodes. Until recently these devices have been used only at or near room temperature and even minute changes in the ambient temperature have caused 
measurable effects on the responsivity of SiAPDs. Heating the photodiode shifts its spectral response curve (including the peak) toward longer wavelengths whilst cooling shifts the curve towards shorter wavelengths (Advanced Optoelectronics, 1990). The following values are typical for the temperature dependence of responsivity:

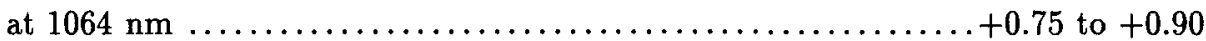
at peak and somewhat below $\ldots \ldots \ldots \ldots \ldots \ldots \ldots \ldots \ldots \ldots \ldots \ldots \ldots \ldots \ldots \ldots . .1$

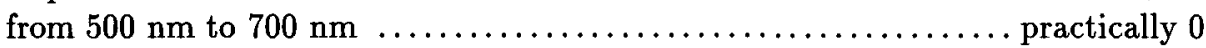

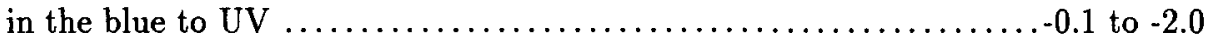

(Advanced Optoelectronics, 1990)

The stronger (and negative) temperature dependence of photodiode responsivity in the UV suggests the low-temperature application of this detectors which is permissible since the operating temperature of SiAPDs ranges from -40 to $+45 \operatorname{deg} C$. Obviously in order to reach high reliability and photometric accuracy the temperature of avalanche photodiodes must be stabilized within 0.1 centigrade. Owing to the compactness of APDs, cooling and thermostatic control can be achieved with a simple Peltier thermoelectric cooler. According to Advanced Photonix Inc., LAAPDs with integral thermoelectric cooling are available on a customised basis.

Thermal stabilization will contribute also to gain uniformity and constancy since the breakdown voltage is also temperature dependent with a coefficient of $+2 \mathrm{~V} / \mathrm{deg}$.

\section{Prospects of further developments of SiAPDs}

In order to get some basis for considerating the future of APDs let us first run through the brief history of PMT development!

1936 Discovery of the principle of the amplification of photoelectrons by means of secondary electron emission in an experimental PMT (Zworykin)

1937 Astronomical testing of an experimental RCA-tube (Kron)

1938 The start of 'mass' production of PMTs (Rajchman)

Complex cathode surfaces (caesium and antimony) $\gg$ fourfold sensitivity (Grlich). The $Q E$ of the new cathodes exceeded 20 times that of the best photoemulsions.

1939/45 WWII: military applications of the tubes, quick development of construction

1946 The introduction of the method of photon counting

1947 The start of the glory of the venerable 1P21 PMT (RCA). Small, compact design, $\mathrm{S} 4$ cathode in the centre of the tube, $Q E$ (at $400 \mathrm{~nm}$ ) $22 \%$, sensitivity range $300 \mathrm{~nm}$ to $650 \mathrm{~nm}, 9$ dynodes, amplification one million, dark current $1 \mathrm{nA}$. 
This is the most extensively used PMT in astronomical photon detection.

1949/55 Observatoire de Paris program: 250 PMTs, up to 20 dynodes, UV, Visible and IR sensitivity, giant tubes and long contacting wires etc.(Lallemand)

1952 Photometric measurements with PMTs in the IR (Hardie)

1953 UBV-system of photoelectric photometry (Johnson \& Morgan)

1957 extended sensitivity range (Ag-O-Cs cathode) up to $1100 \mathrm{~nm}$ (ITT)

1960/70 Wide offer of PMTs by various manufacturers (dimensions, number of dynodes, gain, photocathodes, window materials etc.)

1980- Thermoelectric coolers, commercially available photon counting systems, magnel shielding

1984/85 NO SIGNIFICANT IMPROVEMENTS SINCE THEN! The start of PMTagony?

It is remarkable that astronomers of the thirties were so eager to benefit from the discovery of the PMT and tried to use it immediately. On the other hand it is obvious also that almost nothing happened in the past decade and next to nothing during the preceding two that could have revolutionized PM-theory and/or technology. This definitely suggests that the PMT-era is drawing to a close and in line with that semiconductor technology affects various areas of photodetector applications. My suggestion is to grasp the opportunities of solid-state optoelectronics.

\section{Expectations of SiAPD development during the next 2-3 years}

Having seen the sophistication of avalanche photodiodes explained briefly, the following essential improvements can be expected:

- extension of the spectral sensitivity to the blue-violet-UV range

- enhancement of overall sensitivity, detection of 10-photon and single-photon pulses

- lowering or compensation of the APD's dark current

- substantial increase or multiplication of the avalanche gain by operating the device above the breakdown voltage

- reduction of the LAAPD's price (this is only a desire of the author not of the manufacturers) 
7. Those Fields of astronomy which could benefit soon from the use of APDs

The most important experimental advantages of APDs over PMTs can be summarized as follows:

- extremely high quantum efficiency in the visible-red-NIR spectral range

- unprecedented dynamic range

- compactness, ruggedness, immunity to magnetic fields, no settling time and very good tolerance of overload

Consequently every field of astronomical photometry could benefit from their use especially those which are aimed at the detection of red (low temperature) objects, $\mathrm{R}$ and I colours low-mass stars ( $\mathrm{dK}, \mathrm{dM}$ spectral type and brown dwarfs), eruptive variables (flare stars!), $\mathrm{H} \alpha$ emission objects and highly reddened objects. The use of APDs is very promising in space applications and also in campus observatories because they are astronomer- and student-proof. When the competition between manufacturers results a substantial reduction of their price, APDs should also be the preferred photon detectors for serious amateur photometrists.

\section{Acknowledgements}

This research was partly supported by grant (\# 782/91) from the Hungarian Foundation for Higher Education and Research. I would like to acknowledge the kind help of Advanced Photonix Inc., Advanced Optoelectronics, Hamamatsu Photonics, Princeton Instruments Inc. and THORN EMI Electron Tubes Ltd.

\section{References:}

ADVANCED OPTOELECTRONICS, 1990, Photosensor Product Catalog, p.2.

Advanced Photonix Inc., 1992, Large Area Avalanche Photodiodes, p.2.

Eccles, M.J., Sim, M.E. \& Tritton, K.P., 1983, Low light level detectors in astronomy, p.113.

MacGregor, A., 1991, Photonics Spectra, Vol.bf 25, p.139.

Noble, M., 1991, Lasers \& Optronics, Sept. 1991, Elsevier.

Saloff, D. and Madden, M., 1992, Photonics Spectra, Vol. 26, p.111.

Smith, N., 1993, Poster Papers on Stellar Photometry, IAU Coll. 136, Dublin Inst. for Advanced Studies.

Princeton Instruments Inc., 1991, Charge Coupled Devices - CCD Area Array Detectors, p.2-3.

THORN EMI Electron Tubes Ltd., 1986, Photomultipliers, p.18. 


\section{Discussion}

T.J. Kreidl: Would you please comment on those manufacturers who currently supply the large-cathode APDs and indicate approximate prices? Also, have you taken any astronomical data with any APDs at this time?

Śzécsényi-Nagy: Unfortunately I have no information even about approximate prices of LAAPDs. My data refer to information from Advanced Photonix, Inc., a Xinus Company, but other manufacturers, like Hamamatsu Photonics, offer LAAPDs too, (see the brochure Product Guide which was kindly placed by the organizers of this meeting into our portfolios).

D. Dravins: At Lund Observatory, we have been testing an avalanche photodiode detector in the laboratory, for more than a year. The detectors seem very promising with regard to, for example, ease of use, and quantum efficiency. However, there is still a serious problem in obtaining acceptably low dark count rates with sufficiently large detectors. With our 0.15 mm diameter $A P D$ we get about 50 dark counts per second, which would be acceptable but an astronomical interesting detector would need to be one order of magnitude larger in diameter, and two orders of magnitude greater in area. Since the dark counts seem to increase in proportion to detector area, significant improvements are needed before such systems can be used for faint sources.

Śzécsényi-Nagy: I agree with you. Amongst SAAPDs the newly developed SPCM-200$\mathrm{PQ}$ has been reported with a dark count as low as $2 \mathrm{c} / \mathrm{s}$ (MacGregor. A., 1991), which is significantly lower than yours. For LAAPDs ( 0.5 in diameter) the dark current increases from $60 \mathrm{nA}$ to $200 \mathrm{nA}$ in a reverse bias range of 450 to 2250 volts. The gain of the device over the same range increases from practically 1 to 30 while with a dark current of $500 \mathrm{nA}$ the gain reaches about $250-300$.

M. K. Tsvetkov: I am so happy to hear about the future application of Avalanche Photodiode (APD) detectors in electrophotometry. APD's are based on the discovery of photoelectrets by the Bulgarian professor Georgi Nadjakov, in 1937. He worked at this time in Paris in the Laboratory of Professor Paul Langevin.

Śzécsényi-Nagy: Thank you for your interesting comment.

N. Smith: Using an RCA 30921 APD it is possible to operate in photon counting mode and with active quenching it is linear (to $\sim 1 \%$ ) up to $100 \mathrm{KHz}$. The major drawback is the small active area of approximately $0.25 \mathrm{~mm}^{2}$. Typical dark count rates are $<200 \mathrm{c} / \mathrm{s}$ at $-20^{\circ} \mathrm{C}$. Using light cones it may be possible to increase the effective active area. Afterpulsing and problems with light emitted by the avalanche process in the APD can be overcome by introducing a deadtime of typically 40-100ns.

R.M. Redfern: There seems to be some confusion, in the questions, between APDs operating in the linear and photon-counting 'geiger' mode. Is there any suggestion that these large area disks can be operated in the 'geiger' mode?

Śzécsényi Nagy: Yes, the manufacturer of the LAAPD mentioned here definitely suggests this kind of operation. 